\title{
Evaluation of the human placenta optical scattering properties using continuous wave and frequency-domain diffuse reflectance spectroscopy
}

\author{
Siddharth M. Khare, $,{ }^{a}, \uparrow$ Thien Nguyen $\odot,{ }^{a, \dagger}$ Afrouz A. Anderson, $,{ }^{a}, \uparrow$ \\ Brian Hill, ${ }^{a}$ Roberto Romero,, b,c,d,e,f,g and Amir H. Gandjbakhche ${ }^{a, *}$ \\ ${ }^{a}$ National Institute of Child Health and Human Development, National Institutes of Health, \\ Bethesda, Maryland, United States \\ ${ }^{b}$ U.S. Department of Health and Human Services, Eunice Kennedy Shriver National Institute of \\ Child Health and Human Development, National Institutes of Health, Perinatology Research \\ Branch, Division of Obstetrics and Maternal-Fetal Medicine, Division of Intramural Research, \\ Bethesda, Maryland, and Detroit, Michigan, United States \\ ${ }^{c}$ University of Michigan, Department of Obstetrics and Gynecology, Ann Arbor, Michigan, \\ United States \\ ${ }^{\mathrm{d}}$ Michigan State University, Department of Epidemiology and Biostatistics, East Lansing, \\ Michigan, United States \\ ${ }^{\mathrm{e}}$ Wayne State University, Center for Molecular Medicine and Genetics, Detroit, Michigan, \\ United States \\ ${ }^{\mathrm{f}}$ Detroit Medical Center, Detroit, Michigan, United States \\ ${ }^{g}$ Florida International University, Department of Obstetrics and Gynecology, Miami, Florida, \\ United States
}

\begin{abstract}
Significance: Placenta is an essential organ for fetal development and successful reproduction. Placental insufficiency can lead to fetal hypoxia and, in extreme cases anoxia, leading to fetal death. Of the 145 million deliveries per year worldwide, $\sim 15$ million neonates are small for gestational age and, therefore, at risk for antepartum and intrapartum hypoxia. Clinical methods to assess placental function largely rely on the assessment of fetal heart rate changes but do not assess placental oxygenation. Near-infrared spectroscopy (NIRS) allows non-invasive, real-time assessment of tissue oxygenation in intact organs, which can be used to assess placental oxygenation. However, tissue optical properties can affect the accuracy of methods to measure tissue oxygenation.

Aim: This study was performed to estimate the scattering coefficient of the human placenta. We have computed the scattering coefficients of the human placenta for the range of 659 to $840 \mathrm{~nm}$ using two methods of diffuse reflectance spectroscopy (DRS).

Approach: Measurements were performed using an in-house DRS device and a well-established frequency-domain diffuse optical spectroscopic system (DOSI). Measurements were performed in eight placentas obtained after cesarean deliveries. Placentas were perfused with normal saline to minimize the effects of absorption due to blood. Three sites per placenta were measured. Absorption and scattering coefficients were then calculated from the measured reflectance using the random walk theory for DRS and frequency-domain algorithm for DOSI.

Results: Average reduced scattering coefficient $\left(\mu \mathrm{s}^{\prime}\right)$ was $0.943 \pm 0.015 \mathrm{~mm}^{-1}$ at $760 \mathrm{~nm}$ and $0.831 \pm 0.009 \mathrm{~mm}^{-1}$ at $840 \mathrm{~nm}$, and a power function $\mu \mathrm{s}^{\prime}=1.6619(\lambda / 500 \mathrm{~nm})^{-1.426}$ was derived for the human placental scattering coefficient.

Conclusion: We report for the first time the scattering coefficient of the human placenta. This information can be used to assess baseline scattering and improve measurements of placental oxygen saturation with NIRS.
\end{abstract}

\footnotetext{
*Address all correspondence to Amir H. Gandjbakhche, gandjbaa@mail.nih.gov

${ }^{\dagger}$ These authors have made equal contributions to the manuscript.
} 
(C) The Authors. Published by SPIE under a Creative Commons Attribution 4.0 Unported License. Distribution or reproduction of this work in whole or in part requires full attribution of the original publication, including its DOI. [DOI: 10.1117/1.JBO.25.11.116001]

Keywords: diffuse optical spectroscopic system; near-infrared spectroscopy; power function; random walk theory; scattering coefficient; tissue oxygenation.

Paper 200250LRR received Aug. 4, 2020; accepted for publication Oct. 14, 2020; published online Nov. 5, 2020.

\section{Introduction}

The placenta is an essential organ for fetal development and successful pregnancy. ${ }^{1}$ The villous tree of this organ provides an interface between the maternal and fetal circulations. ${ }^{1}$ The respiratory function of the placenta consists of providing oxygen from maternal blood to the fetus, and carbon dioxide from the fetus into the maternal circulation. ${ }^{2}$ Other important functions of the placenta include providing nutrients to maintain fetal growth, waste removal, endocrine, and immunological functions. ${ }^{1}$

The term "placental insufficiency" is used in clinical obstetrics to refer to a state in which the organ fails to deliver nutrients or oxygen, which may lead to fetal growth restriction or, in extreme cases, death from anoxia. ${ }^{3-6}$ Most tests of placental function rely on indirect parameters, such as the assessment of fetal growth, Doppler assessment of vascular impedance in the maternal or fetal circulation, or the determination of placental products such as human chorionic gonadotropin, human placental lactogen, placental-specific protein 13, or other products manufactured by the organ. ${ }^{7}$ Given the importance of placental respiratory function for fetal survival, a method to assess placental oxygenation in real time would be of considerable importance in clinical medicine, and in the understanding of the pathophysiology of pregnancy.

Tissue oxygenation can be measured in-vivo by calculating the tissue oxygenation index (TOI). Blood oxygen saturation is now routinely used in clinical medicine to assess respiratory function and is part of routine practice. The most common method to assess oxygenation is using optical methodologies, which largely rely on diffuse reflectance spectroscopy (DRS). ${ }^{8-10}$ Typically, for this purpose, light in the near-infrared (NIR) range (700 to $900 \mathrm{~nm}$ ) of wavelengths is directed to a tissue in close proximity to the circulation. Given that NIR can penetrate tissue without substantial attenuation by normal components, such as adipose tissue and water, light can diffuse into the tissue from a source and backscattered light can be detected with optical methods. The principle behind this technology consists of the detection of changes in the concentrations of oxyhemoglobin ( $\mathrm{HbO})$ and deoxyhemoglobin.

To reach the placenta in-vivo, the light needs to pass through the skin, adipose tissue, and myometrium (uterus). In another study on 12 pregnant women in the third trimester, we found the total thickness of these tissues ranged from 7.1 to $42.5 \mathrm{~mm}$ (unpublished data). Near-infrared spectroscopy (NIRS) can probe depths of up to $30-\mathrm{mm}$ below the skin surface. This was determined using Monte Carlo photon diffusion simulations of a three-layer tissue (skin, fat, and muscle) using the hop-drop-spin method described by Steve Jacques (data not yet published). Therefore, we propose that NIRS will be able to noninvasively probe the upper few millimeters of the placenta in a pregnant woman with total maternal tissue thickness $<30$-mm and with the placenta positioned in the anterior, fundal, and side of the uterus.

There have been very few studies on placental oxygenation. ${ }^{11-13}$ Matsuo et al. ${ }^{11}$ reported that placental oxygenation is lower in preeclampsia, measured by the umbilical arterial-venous oxygen difference. Hasegawa et al. used an NIRS device to perform transabdominal measurements of placental TOI in pregnancies complicated with a small for gestational age fetus. They reported higher placental TOI values for fetuses whose mothers had preeclampsia and placental abnormalities, whereas those with umbilical cord abnormalities showed a lower placental TOI values. ${ }^{12}$ Moreover, the authors proposed that a high TOI represented a greater than normal concentration of $\mathrm{HbO}$, which resulted from a reduced oxygen exchange caused by placental pathology in the intervillous space. Kakogawa et al. ${ }^{13}$ also reported that the NIRS probe can 
be utilized to assess placental oxygenation. However, these studies have not taken into account sufficient information about the optical scattering of the placenta.

Because both scattering and absorption contribute to the light attenuation in the tissue and the soft tissue is in general a highly scattering medium, incorporating scattering coefficient of placental tissue can improve the reliability of the assessment of tissue oxygenation. Currently, the scattering coefficient of the human placental tissue is unknown. Herein, we report the effective/ reduced scattering coefficient $\left(\mu \mathrm{s}^{\prime}\right)$ in ex-vivo placental tissue samples.

\section{Materials and Methods}

\subsection{Measurement Devices}

We chose two devices to measure placental optical properties: (1) an in-house continuous-wave (CW) system, featuring two wavelengths. This device records spatially resolved diffused reflectance with source-detector (SD) separation from 7.4 to $54.4 \mathrm{~mm}$ and (2) a frequency-domain diffuse optical spectroscopic system (DOSI) with a fixed SD separation of $28 \mathrm{~mm}$.

\subsubsection{Continuous-wave spatially diffuse reflectance spectroscopy}

Figure 1 shows the in-house DRS device, which consists of a 16-element photodiode array (S4111-16R with driver circuit C9004, Hamamatsu Photonics K.K., Hamamatsu City, Shizuoka, Japan) as a detector and three dual-wavelength NIR light-emitting diodes (LEDs; L760/84005A, Ushio Inc., Tokyo, Japan) as the light sources. The center-to-center distance between consecutive elements in the photodiode array is $1 \mathrm{~mm}$. The LEDs emit light at 760 and $840 \mathrm{~nm}$ and are placed at distances of 7.4, 23.4, and $39.4 \mathrm{~mm}$ from the first element of the photodiode array. The system was custom-built for high-speed semi-automated data acquisition and storage. Data were acquired with 10-bit resolution. A frame to hold the light sources and detector was 3Dprinted to make the assembly easy to handle. It was sealed with silicone glue to prevent moisture from entering the device. The system can measure a diffuse reflectance profile with 1-mm spatial resolution in the SD distance range from 7.4 to $54.4 \mathrm{~mm}$. The LEDs were designed to emit light at four different intensity levels, each measured with a power meter (S120C, Thorlabs, New Jersey) to compensate for the LED-to-LED variations. The detection performance of all 16 photodiode elements was measured. The differences among the elements were negligible and have not been accounted for herein.

\subsubsection{Frequency-domain diffuse optical spectroscopic system measurements}

The frequency-domain system, also called the DOSI, is well-characterized and has been previously described. ${ }^{14,15}$ Measurements were performed using a hand-held probe with a fixed SD distance of $28 \mathrm{~mm}$. Amplitude-modulated near-infrared light at four wavelengths $(659,689,781$, and $829 \mathrm{~nm}$ ) were utilized for frequency-domain measurements; the broadband continuous wave mode of the device was not enabled. The modulated frequency of DOSI ranges from 40 to $400 \mathrm{MHz}$, with a source power of all four wavelengths of $20 \mathrm{~mW}$. The detector is an avalanche photodiode with 1.5-mm diameter (Hamamatsu Photonics K.K., Hamamatsu City, Shizuoka,

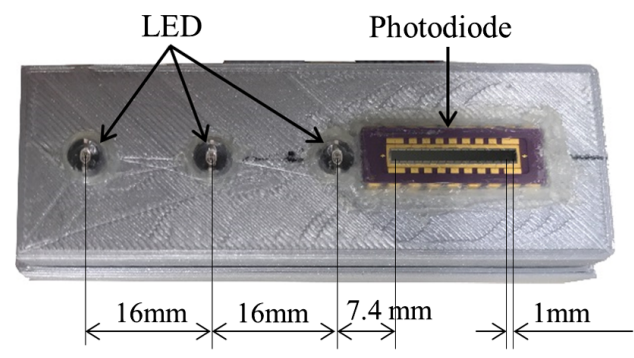

Fig. 1 DRS device built in-house for measurement of the optical properties of the placental tissue. 

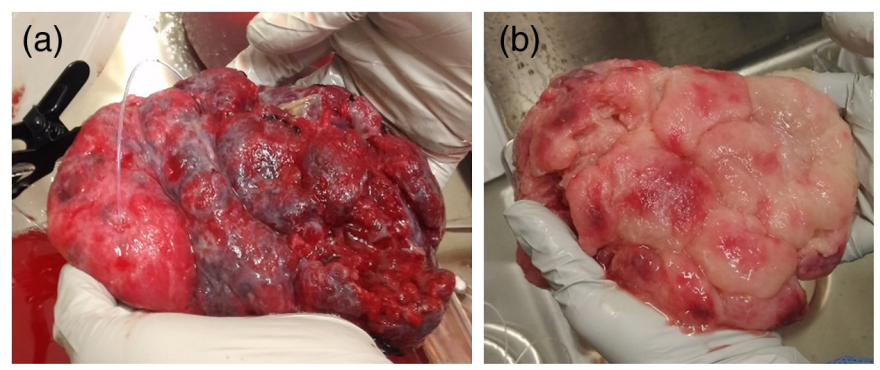

Fig. 2 Placental tissue (a) during perfusion of the maternal lobe. Removal of blood can be observed in the lighter color of the lobe on the left, and (b) after perfusion of all maternal lobes.

Japan). Details of the system calibration and algorithms used in this system to measure absorption and reduced scattering coefficients have been reported. ${ }^{14-16}$

\subsection{Placental Tissue Preparation}

Eight normal placentas (gestational age: 37 to 41 weeks) were obtained from cesarean deliveries with pregnancies without complication at Walter Reed Hospital (Bethesda, Maryland). The maternal age was between 18 and 40 years old. This study excluded all placentas from mother with major fetal congenital anomalies, maternal hypertension/hypertensive disorder, diagnosis of intraamniotic infection, diagnosis of intrauterine growth restriction, maternal diabetes, hypertension, multiple gestation, and placental abnormalities. In addition, placentas that required pathological analysis were excluded. The approximate thickness of the placental tissues was $25 \mathrm{~mm}$. All placentas were delivered to the laboratory within $30 \mathrm{~min}$ of the procedure. The whole placentas were perfused with saline to remove the blood for accurate assessment of the reduced scattering coefficient. The time-lapse between receiving the placenta and the onset of perfusion was about $6 \mathrm{~min}$. The umbilical vein was cannulated, and the placenta was perfused with a phosphate-buffered saline $1 \times$ solution using a peristaltic pump at the flow rate of $100 \mathrm{ml} / \mathrm{min}$ for about $30 \mathrm{~min}$. After perfusion of the fetal side, the maternal side was perfused for about 45 to 60 min by inserting thin Teflon tubes at multiple locations in the maternal lobes. The flow rate from each tube was $\sim 10 \mathrm{ml} / \mathrm{min}$. The DRS device was utilized to measure optical properties at multiple placental locations. This study was approved by the National Institutes of Health and the Walter Reed National Military Medical Center (Bethesda, Maryland). Figure 2 shows the maternal side of the placenta before and after perfusion, respectively.

\subsection{Estimation of Reduced Scattering Coefficient $\left(\mu S^{\prime}\right)$ and Absorption Coefficient ( $\mu$ a)}

The random walk theory for diffusion of photons through an absorbing and scattering medium has been previously described. ${ }^{17}$ For a semi-infinite medium, the probability that a photon is diffusely reflected to the surface of the medium at a distance $r$ from the source is given by Eq. (1), and $\mu \mathrm{a}$ and $\mu \mathrm{s}^{\prime}$ are the absorption and reduced scattering coefficients, respectively.

$$
\Gamma(r)=\frac{e^{-2 \frac{\mu_{a}}{\mu_{s}^{\prime}}}}{4 \pi \frac{\mu_{s}^{\prime} r}{\sqrt{2}}}\left[e^{-r \sqrt{3 \mu_{a} \mu_{s}^{\prime}}}-e^{\left(-\sqrt{6 \frac{\mu_{a}}{\mu_{s}^{\prime}}\left(\frac{\mu_{s}^{\prime 2} r^{2}}{2}+4\right)}\right)}\right] .
$$

The intensity of light detected at the surface depends on the photon flux that, in turn, depends on the characteristics of the light source and detection efficiency. In our device, the intensity is measured in terms of the photodiode count (range: 0 to 1023). As a result, the intensity values are expressed in arbitrary units. To account for the source intensity, we calculated the intensity $I(r)$ at distance $r$ from the source as

$$
I(r)=\alpha \Gamma(r) .
$$


Khare et al.: Evaluation of the human placenta optical scattering properties using continuous wave...

Table 1 Scattering coefficients of the three phantoms used for validation.

\begin{tabular}{|c|c|c|c|c|c|}
\hline \multirow[b]{2}{*}{ Phantom name } & \multirow[b]{2}{*}{ Source of $\mu \mathrm{s}^{\prime}$ values } & \multicolumn{2}{|c|}{$\mu \mathrm{s}^{\prime}$ at $760 \mathrm{~nm}\left(\mathrm{~mm}^{-1}\right)$} & \multicolumn{2}{|c|}{$\mu \mathrm{s}^{\prime}$ at $840 \mathrm{~nm}\left(\mathrm{~mm}^{-1}\right)$} \\
\hline & & Standard & DRS & Standard & DRS \\
\hline fNIR devices LLC & Provided by the company & 1.06 & 0.93 & 0.96 & 0.85 \\
\hline Acrin7 & Measured using DOSI & 0.66 & 0.70 & 0.57 & 0.60 \\
\hline Scrooge & Measured using DOSI & 0.91 & 0.88 & 0.82 & 0.78 \\
\hline
\end{tabular}

The constant $\alpha$ is the instrument calibration factor that corresponds to the source intensity. To determine $\alpha$, we employed a reference phantom made of Polydimethylsiloxane (PDMS), TiO2 powder, and India ink. The optical properties $\mu$ a and $\mu \mathrm{s}^{\prime}$ of this phantom were determined using a well-characterized frequency-domain system (DOSI at Beckman laser institute, University of California, Irvine in Irvine, California) and reconfirmed by a commercial time-domain system (Hamamatsu Photonics K.K., Hamamatsu City, Shizuoka, Japan), $\mu \mathrm{a}=0.0052 \pm 0.0001 \mathrm{~mm}^{-1}$ and $\mu \mathrm{s}^{\prime}=0.6914 \pm 0.0083 \mathrm{~mm}^{-1}$ at $760 \mathrm{~nm}$ and $\mu \mathrm{a}=0.0036 \pm 0.0001 \mathrm{~mm}^{-1}$ and $\mu \mathrm{s}^{\prime}=$ $0.6148 \pm 0.0095 \mathrm{~mm}^{-1}$ at $840 \mathrm{~nm}$. These values were substituted in the random walk Eq. (1) to obtain the probability of the diffuse reflectance $\Gamma(r)$. Next, the in-house DRS device was used on the reference phantom to record the reflection profile $I(r)$ at all possible SD distances $r$. From Eq. (2), the instrument calibration factor $\alpha$ was calculated using the measured intensity values and the derived $\Gamma(r)$ values.

Equation (2), with the known $\alpha$ at 760 and $840 \mathrm{~nm}$, was then applied to calculate the optical properties using a least-square fit to the data measured by the DRS device. The least-square fitting was performed utilizing the Matlab ${ }^{\circledR}$ software (The MathWorks ${ }^{\circledR}$ Inc, Massachusetts) Curve Fitting Tool (95\% confidence bounds). The first 16 SD separations were included in our analysis as the larger SD separation yielded insufficient intensities. This methodology was validated using three phantoms, whose optical properties at four different wavelengths were provided by either the company (fNIR Devices LLC, New Orleans, Louisiana, 680, 750, 780, and $810 \mathrm{~nm}$ ) or measured with a DOSI system and was confirmed with the time domain system measurement. Since the provided wavelengths were different with the wavelengths used in our DRS device, we could only compute $\mu \mathrm{s}^{\prime}$ (not $\mu \mathrm{a}$ ) at 760 and $840 \mathrm{~nm}$ by a power function fitting (Table 1). The $\mu \mathrm{s}^{\prime}$ values obtained using the DRS devices have an average of 7.2\% deviation from the provided/FD-measured values.

The above method was then applied to the DRS intensity data from the perfused placentas to obtain their optical properties.

\section{Results}

\subsection{Random Walk Theory Fitting}

The diffuse reflectance profile was measured on the maternal side of the perfused placenta. The small amount of blood that remains after perfusion within the tissue is expected to absorb the photons without changing the optical scattering of the tissue significantly. Figure 3 shows representative diffuse reflectance profiles measured utilizing the in-house DRS system on the maternal side of a perfused placenta. The random walk model fits well to the DRS profiles. The goodness of the fit was as follows: $R^{2}=0.996$ and RMSE $=18.26$ at $760 \mathrm{~nm}$ and $R^{2}=0.997$ and RMSE $=8.135$ at $840 \mathrm{~nm}$. The instrument calibration factors, calculated as described in Sec. 2.3, were 1140261 at $760 \mathrm{~nm}$ and 902105 at $840 \mathrm{~nm}$.

\subsection{Absorption and Reduced Scattering Coefficients Derived from the CW DRS Device}

For each placenta, the measurements and calculations were performed at three sites on the maternal side with each region being measured three times. The reduced scattering $\left(\mu \mathrm{s}^{\prime}\right)$ and 
Khare et al.: Evaluation of the human placenta optical scattering properties using continuous wave...
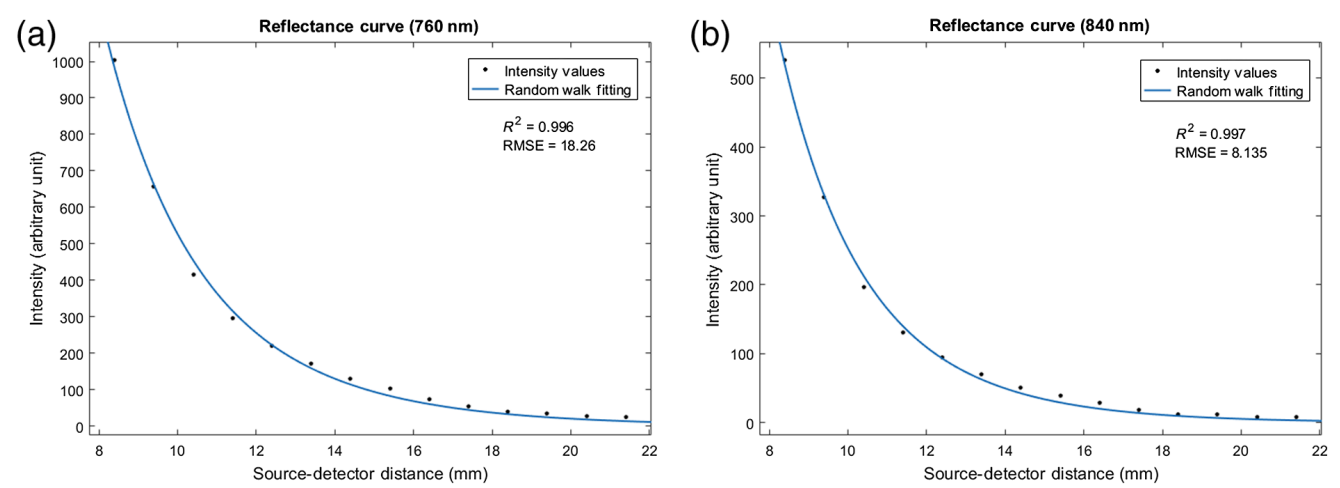

Fig. 3 Diffuse reflectance profiles at the maternal side of a perfused placenta: Measured intensity values (black dots) plotted as a function of SD distance and random walk fit (blue solid line) at (a) 760 and (b) $840 \mathrm{~nm}$.

Table 2 Mean and standard errors of reduced scattering ( $\left.\mu s^{\prime}\right)$ and absorption ( $\left.\mu \mathrm{a}\right)$ coefficients of seven perfused placental tissues.

\begin{tabular}{lcccc}
\hline \hline Placenta & $\mu \mathrm{s}^{\prime}(760)\left(\mathrm{mm}^{-1}\right)$ & $\mu \mathrm{a}(760)\left(\mathrm{mm}^{-1}\right)$ & $\mu \mathrm{s}^{\prime}(840)\left(\mathrm{mm}^{-1}\right)$ & $\mu \mathrm{a}(840)\left(\mathrm{mm}^{-1}\right)$ \\
\hline 1 & $0.924( \pm 0.014)$ & $0.005( \pm 0.003)$ & $0.798( \pm 0.011)$ & $0.006( \pm 0.003)$ \\
2 & $0.822( \pm 0.004)$ & $0.008( \pm 0.0001)$ & $0.721( \pm 0.004)$ & $0.005( \pm 0.0001)$ \\
3 & $1.055( \pm 0.020)$ & $0.018( \pm 0.002)$ & $0.928( \pm 0.009)$ & $0.013( \pm 0.0009)$ \\
4 & $1.020( \pm 0.012)$ & $0.015( \pm 0.001)$ & $0.903( \pm 0.009)$ & $0.006( \pm 0.0005)$ \\
5 & $0.900( \pm 0.023)$ & $0.015( \pm 0.004)$ & $0.787( \pm 0.011)$ & $0.007( \pm 0.002)$ \\
6 & $0.899( \pm 0.013)$ & $0.008( \pm 0.0009)$ & $0.821( \pm 0.007)$ & $0.004( \pm 0.0004)$ \\
7 & $0.979( \pm 0.021)$ & $0.017( \pm 0.001)$ & $0.859( \pm 0.010)$ & $0.005( \pm 0.001)$ \\
Mean & $0.943( \pm 0.015)$ & $0.012( \pm 0.002)$ & $0.831( \pm 0.009)$ & $0.007( \pm 0.001)$ \\
\hline \hline
\end{tabular}

absorption $(\mu \mathrm{a})$ coefficients of a placenta were then obtained by averaging data from nine measurements for each placenta. Data from one of eight placentas were excluded because of the low measured intensity. Reduced scattering $\left(\mu \mathrm{s}^{\prime}\right)$ and absorption $(\mu \mathrm{a})$ coefficients of seven perfused placental tissues are given in Table 2. The mean reduced scattering and absorption coefficients of perfused placental tissue are $\mu \mathrm{s}^{\prime}=0.943 \pm 0.015\left(\mathrm{~mm}^{-1}\right)$ and $\mu \mathrm{a}=0.012 \pm 0.002\left(\mathrm{~mm}^{-1}\right)$ at $760 \mathrm{~nm}$ and $\mu \mathrm{s}^{\prime}=0.831 \pm 0.009\left(\mathrm{~mm}^{-1}\right)$ and $\mu \mathrm{a}=0.007 \pm 0.001\left(\mathrm{~mm}^{-1}\right)$ at $840 \mathrm{~nm}$. The $\mu \mathrm{s}^{\prime}$ values did not vary drastically despite the observed changes in the $\mu$ a values (Table 2).

\subsection{Reduced Scattering Coefficients Measured with the Frequency-Domain System}

The DOSI system provided optical property measurements at four wavelengths, hence it was used to measure the reduced scattering coefficients of one of the placentas (placenta 1, Table 2) and compared to the coefficients estimated with the DRS device. Figure 4 shows amplitude and phase model fits to the measured data on placenta 1 using the DOSI system. The measurement using the DOSI system yielded $\mu \mathrm{s}^{\prime}=1.129 \pm 0.025 \mathrm{~mm}^{-1}$ at $659 \mathrm{~nm}, \mu \mathrm{s}^{\prime}=1.050 \pm$ $0.021 \mathrm{~mm}^{-1}$ at $686 \mathrm{~nm}, \mu \mathrm{s}^{\prime}=0.866 \pm 0.019 \mathrm{~mm}^{-1}$ at $787 \mathrm{~nm}$, and $\mu \mathrm{s}^{\prime}=0.810 \pm 0.015 \mathrm{~mm}^{-1}$ at $831 \mathrm{~nm}$. A power function $\mu_{\mathrm{s}}^{\prime}=a(\lambda / 500 \mathrm{~nm})^{-b}$ was fitted to these reduced scattering coefficients with $a=1.6619$ and $b=1.426$ with $R^{2}=0.9978$ (Fig. 5). The reduced scattering coefficients at 760 and $840 \mathrm{~nm}$ were then derived as $\mu \mathrm{s}^{\prime}=0.915$ and $0.793 \mathrm{~mm}^{-1}$, respectively. 
Khare et al.: Evaluation of the human placenta optical scattering properties using continuous wave...
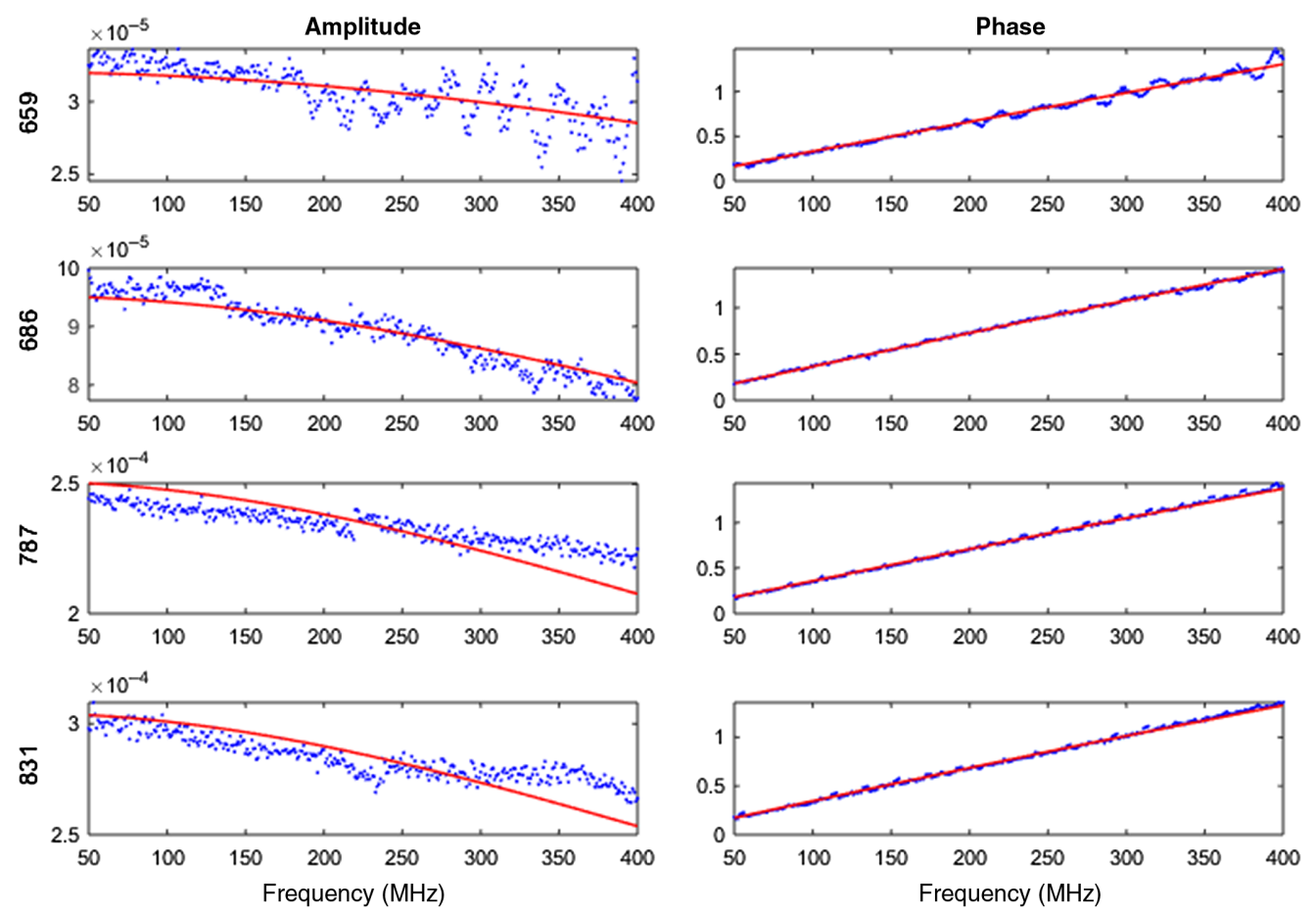

Fig. 4 Amplitude and phase model fit of DOSI data measured on placenta 1

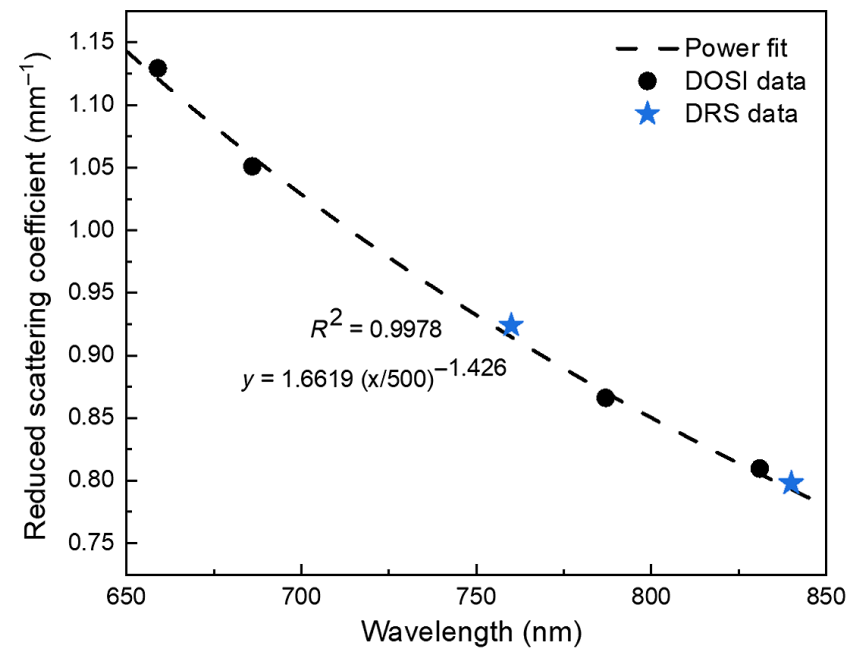

Fig. 5 Reduced scattering coefficients calculated from two devices. The black dashed line shows the power fit of the DOSI measurement (black dots) while the blue dots represent the DRS measurements from placenta 1. Abbreviations: DOSI, diffuse optical spectroscopic system; DRS, diffuse reflectance spectroscopy.

\section{Discussion}

In this study, we have shown the feasibility of using a continuous wave DRS system in assessment of reduced scattering coefficient of the placental tissue ex-vivo. However, it is important to emphasize that the measurement in this study was performed on well-perfused placenta tissues. Based on the study by Yao et al., ${ }^{18}$ the placental blood volume fraction, when the umbilical cord was clamped $5 \mathrm{~s}$ after delivery was $\sim 21 \%$. The removal of the blood from these placentas affects the measured reduced scattering coefficient from its value in-vivo. However, the residual blood volume varies by large amounts in the ex-vivo placentas. Placental blood is lost during the 
cesarean delivery, transportation of the placenta and the umbilical cord clamping time. According to Yao et al., ${ }^{18}$ clamping time can change the residual placental blood volume up to $60 \%$. In addition, even though the umbilical cord was clamped, the blood inside of the placenta still drained out from the maternal side during the transportation to the lab. Therefore, we report the reduced scattering coefficient of the underlying placental tissue without blood. Scattering properties of the blood will need to be considered when performing noninvasive monitoring of placental oxygenation with known or estimated placental blood volume fraction.

Additionally, the results from the in-house DRS device can be affected to some extent by the light reflected back into the tissue from the boundary between the tissue surface and the device. However, the difference in the DRS intensity was negligible $(<1 \%)$ when measurements were performed on the Acrin phantom with and without coating the surface of the device using a black tape. Hence, we expect the effect of reflected light on calculated scattering coefficient is insignificant. In addition to reporting the optical scattering of the human placenta, this work provides a simpler, more compact, and cost-effective system and methodology to measure the scattering properties of the human placental tissue ex-vivo. These factors will help in generating a large database from normal and abnormal pregnancies. The data presented here act as a reference point for future studies and will help provide more accurate distribution of the optical scattering properties of the human placenta.

\section{Conclusion}

In this study, we used DRS in continuous wave and frequency-domain mode to measure the optical scattering coefficient of the human placenta without blood in the NIR range. The provided power law equation can be used to calculate the reduced scattering coefficient at any wavelength in the NIR band. This platform can facilitate the study of key parameters such as tissue oxygenation and blood flow within the placenta. The results reported herein could allow a more precise calculation of placental oxygenation and assess the respiratory function of the organ noninvasively. In the future, it would be important to assess the scattering properties of placentas obtained from normal pregnancy and those with complications. Overall, our in-house DRS device provides a simpler and compact means for measurement of optical properties on the placenta, which can help generate larger database.

\section{Disclosures}

We declare that there is neither actual nor potential conflict of interest related to this manuscript.

\section{Acknowledgments}

We would like to thank Dr. Robert Walton at Walter Reed Hospital for providing the placentas. This research was supported by the Intramural Research Grant in Eunice Kennedy Shriver National Institute of Child Health and Human Development, the Perinatology Research Branch, Division of Obstetrics and Maternal-Fetal Medicine, Division of Intramural Research, National Institutes of Health, U.S. Department of Health and Human Services (NICHD/NIH/DHHS); and with Federal funds from NICHD/NIH/DHHS under Contract No. HHSN275201300006C. Dr. Romero has contributed to this work as part of his official duties as an employee of the United States Federal Government.

\section{References}

1. L. Belkacemi et al., "Maternal undernutrition influences placental-fetal development," Biol. Reprod. 83(3), 325-331 (2010).

2. E. P. Hill, G. G. Power, and L. D. Longo, "A mathematical model of carbon dioxide transfer in the placenta and its interaction with oxygen," Am. J. Physiol. 224(2), 283-299 (1973). 
3. J. M. Browne, "Placental insufficiency," Scottish Med. J. 8(12), 459-465 (1963).

4. G. D. Braunstein et al., "Interrelationships of human chorionic gonadotropin, human placental lactogen, and pregnancy-specific $\beta 1$-glycoprotein throughout normal human gestation," Am. J. Obstet. Gynecol. 138(8), 1205-1213 (1980).

5. U. Krishna and S. Bhalerao, "Placental insufficiency and fetal growth restriction," J. Obstet. Gynecol. India 61(5), 505-511 (2011).

6. B. Huppertz, "Oxygenation of the placenta and its role in pre-eclampsia," Pregnancy Hypertens. 4(3), 244-245 (2014).

7. M. Lopes, "Managing costs in high-risk obstetrics," Am. J. Managed Care 2, 1-8 (2013).

8. A. Afshari et al., "Facial plethora: modern technology for quantifying an ancient clinical sign and its use in cushing syndrome," J. Clin. Endocrinol. Metab. 100(10), 3928-3933 (2015).

9. D. W. Hattery et al., "Diffuse reflectance spectroscopy to quantify inflammation of the oral epithelium in vivo," Proc. SPIE 4613, 59-70 (2002).

10. A. L. Companion, "Theory and applications of diffuse reflectance spectroscopy," in Developments in Applied Spectroscopy, E. N. Davis, Ed., pp. 221-234, Springer, Boston, MA (1965).

11. K. Matsuo et al., "Decreased placental oxygenation capacity in pre-eclampsia: clinical application of a novel index of placental function preformed at the time of delivery," J. Perinat. Med. 37(6), 657-661 (2009).

12. J. Hasegawa et al., "Evaluation of placental function using near infrared spectroscopy during fetal growth restriction," J. Perinat. Med. 38(1), 29-32 (2010).

13. J. Kakogawa et al., "Transabdominal measurement of oxygenation of the placenta by nearinfrared spectroscopy," Semin. Thromb. Hemost. 31(3), 297-301 (2005).

14. K. S. No et al., "Design and testing of a miniature broadband frequency domain photon migration instrument," J. Biomed. Opt. 13(5), 050509 (2008).

15. B. J. Tromberg et al., "Predicting responses to neoadjuvant chemotherapy in breast cancer: ACRIN 6691 trial of diffuse optical spectroscopic imaging," Cancer Res. 76(20), 5933-5944 (2016).

16. H. M. Peterson et al., "In vivo noninvasive functional measurements of bone sarcoma using diffuse optical spectroscopic imaging," J. Biomed. Opt. 22(12), 121612 (2017).

17. A. H. Gandjbakhche and G. H. Weiss, "V: random walk and diffusion-like models of photon migration in turbid media," in Progress in Optics, E. Wolf, Ed., pp. 333-402, Elsevier, North-Holland, Amsterdam (1995).

18. A. C. Yao, J. Lind, and M. Moinian, "Distribution of blood between infant and placenta after birth," Lancet 294, 871-873 (1969).

Siddharth Khare is currently senior research engineer at Micelio Labs, India. He received his MSc degree in physics from the University of Pune in 2009 and his $\mathrm{PhD}$ in physics from Indian Institute of Science in 2016. He did postdoctoral research at the National Institutes of Health from 2017 to 2020 . He has keen interest in applied and interdisciplinary research, especially in the field of biomedical instrumentation and imaging.

Thien Nguyen is currently a postdoctoral fellow at the National Institutes of Health, USA. She received her BS degree in biomedical engineering from Hanoi University of Science and Technology in 2013, and MS and PhD degrees in biomedical science and engineering from Gwangju Institute of Science and Technology in 2015 and 2019, respectively. Her current research interests include optical applications in brain activity and tissue oxygenation.

Afrouz Anderson received her $\mathrm{PhD}$ in biomedical engineering focusing on translational biophotonics from the University of California, Davis, and National Institutes of Health. Her research focuses on the application of noninvasive optical imaging for tissue characterization as well as quantifying brain hemodynamics in neurodevelopmental disorders. Her main areas of research are neurocognitive function in urea cycle disorders and chromosomal disorders and the development of NIRS based methodology for noninvasive tissue monitoring such as placental oxygenation. 
Khare et al.: Evaluation of the human placenta optical scattering properties using continuous wave...

Amir Gandjbakhche is a senior investigator at the National Institutes of Health and head of the Section on Translational Biophotonics. He obtained his PhD in physics with a biomedical engineering specialty from the University of Paris in 1989. He is a fellow of SPIE. His areas of interest are the use of NIRS/EEG to apply to developmental disorders and diseases and using spectroscopic methods to quantify oxygenation in placenta.

Biographies of the other authors are not available. 